\title{
Editorial
}

\section{Siete visiones sobre el presente, pasado y futuro del Boletín de la Sociedad Geológica Mexicana}

\section{Página Contribución}

iv El Boletín de la Sociedad Geológica Mexicana, espejo de la evolución de las Ciencias de la Tierra en México Thierry Calmus

vi Breve historia del Boletín de la Sociedad Geológica Mexicana Elena Centeno García

viii Carta del Presidente de la Sociedad Geológica Mexicana José Antonio Escalera Alcocer

ix 110 años del Boletín de la Sociedad Geológica Mexicana Dante J. Morán Zenteno

xi El Boletín de la Sociedad Geológica Mexicana como revista científica Ángel F. Nieto Samaniego

xiii Mis experiencias e impresiones sobre el desarrollo académico del Boletín de la Sociedad Geológica Mexicana Fernando Ortega Gutiérrez

xiv Indicadores de producción e impacto del Boletín de la Sociedad Geológica Mexicana 
El Boletín de la Sociedad Geológica Mexicana, espejo de la evolución de las Ciencias de la Tierra en México

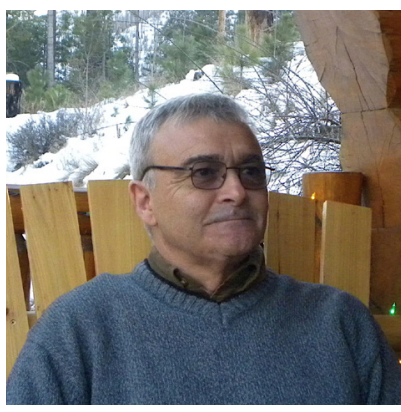

Thierry Calmus

El Dr. Thierry Calmus es Investigador Titular C de la Estación Regional del Noroeste del Instituto de Geología de la Universidad Nacional Autónoma de México, y Co-Editor en Jefe de la Revista Mexicana de Ciencias Geológicas.

Al abrir el portal del Boletín de la Sociedad Geológica Mexicana, un lector curioso no puede dejar de abrir algunos números contemporáneos de los primeros pasos de la Sociedad. El primer número muestra ya la vocación multidisciplinaria que caracteriza las Ciencias de la Tierra: cuenta con dos libretos-guía de excursiones, una reseña de la evolución de la geología en México, simbólico del punto de partida de la Sociedad, cinco artículos relacionados con la geología económica, un artículo de paleontología y uno de volcanología-petrografía. 4 de 10 artículos tienen el mismo autor, el sub-director del Instituto Geológico Nacional. No quiero imaginar los comentarios de la comunidad geológica del siglo XXI al darse cuenta que un funcionario o director publique 4 artículos en un mismo número. $O$ tempora, o mores. Sin embargo, debemos reconocer la fe y el entusiasmo de los fundadores y actores de una comunidad científica balbuciente, marcada por una tradición minera, mas no geológica.

Esta proporción entre el número de artículos dedicados a la Geología Económica y a la Paleontología cambió con el tiempo y se invirtió parcialmente pero, finalmente, ambas áreas representan las dos temáticas fundamentales del Boletín. De los 736 artículos publicados en 112 números desde 1904, 129 son de Paleontología, mientras que 96 se refieren a la Geología Económica, la mayor parte sobre la descripción de depósitos minerales, más que sobre los procesos metalogenéticos. Ambos grupos representan 225 artículos, es decir $30.57 \%$ del total de artículos publicados en 111 años. El análisis de la evolución de la producción de artículos está sesgado debido a dos factores principales: el primero está relacionado con las 4 interrupciones de la publicación, la primera, la más larga, entre 1912 y 1936, la segunda entre 1946 y 1948, la tercera entre 1968 y 1972, y la cuarta entre 1996 y 2000 . El segundo factor que produce un sesgo estadístico corresponde a los números temáticos que amplifican bruscamente la cantidad de artículos, lo cual favorece la representatividad de los temas que fueron objetos de números especiales. Sin embargo, podemos concluir que la Paleontología es la temática dominante a lo largo de la historia del Boletín, lo cual refleja una tendencia de la investigación geológica en México. Observamos incluso un aumento sustancial después de 2000 con 83 artículos en 32 números, ciertamente gracias a la publicación de 44 artículos en solo 3 números especiales, mientras que hubo 37 artículos en 80 números entre 1904 y 1999. Habrá que analizar estos números a detalle en un contexto internacional, en el cual la producción de artículos de Paleontología muestra una tendencia opuesta, y tiende a bajar en las revistas de carácter general, como lo son las revistas de Sociedades Geológicas en el mundo.

Estas primeras observaciones llevan pues, a mi juicio, a dos reflexiones preliminares sobre el Boletín y el contexto nacional: 1) la peculiaridad de la Paleontología en México, inicialmente conforme a una tendencia y una tradición universal hasta la primera mitad del siglo $\mathrm{XX}$, que refleja la importancia de la Paleontología en el conocimiento de la evolución geológica de un país; y posteriormente su permanencia como preponderante en el Boletín, gracias probablemente a una comunidad nacional muy activa y una riqueza paleontológica en el país que lo justifica; 2) una tradición nacional en un país minero, en el cual la geología se concibió mucho tiempo como una herramienta de la exploración y de la explotación minera, y no como una ciencia natural. ¿Valdrá la pena recordar que, en muchas universidades del país, los geólogos son todavía formados como ingenieros y no como científicos?

Habría mucho más que decir a partir de las estadísticas de la producción científica del Boletín. Solamente haré algunas observaciones. Pero antes, cabe aclarar que la clasificación de los artículos, la cual se hizo a partir del solo título, puede ser sujeta a discrepancia y discusión. Un estudio del granito Esperanza del Complejo Acatlán, ¿es un artículo sobre metamorfismo, geología regional, tectónica, geoquímica, petrografía? El título no siempre permite categorizar con precisión $\mathrm{y}$, en este breve trabajo, seguramente existen aproximaciones o errores que se podrían corregir con un análisis más detallado de los contenidos. Sin embargo, llaman la atención algunos resultados. En primero es la ausencia de trabajos relacionados con la Tectónica de Placas, particularmente en los años 60's y 70's cuando, a nivel internacional, se multiplicaron de manera exponencial los trabajos relacionados con esta temática tan prolífica. Entre 1950 y 1966 no se publicó un solo artículo de Tectónica en el Boletín. 9 años después, la aparición de la Revista del Instituto de Geología de la UNAM en 1975 (hecho poco común en la historia de la edición científica, siendo las 
publicaciones científicas tarea de las sociedades científicas y no de las universidades) creó una competencia "desleal", al momento de acaparar una cantidad importante de trabajos del propio Instituto, el cual concentraba la mayor parte de los investigadores en Geociencias del país. Sin embargo, la Tectónica de Placas no aparece tampoco como tal en la Revista del Instituto de Geología.

Como era de esperarse, después de la Paleontología y la Geología Económica, la Estratigrafía ocupa el tercer lugar con 69 artículos (9.4\% del total). Sigue en este orden la Tectónica (41), la Geología Aplicada e Ingeniería (32), la Hidrogeología y Hidrogeoquímica (32), la Geomorfología y Procesos Superficiales (30), la Geología Regional (28), aclarando que este rubro está constituido de artículos en los cuales la región de estudio es el tema principal, la Mineralogía (26), la Petrología Sedimentaria y
Sedimentología (20), la Petrología Ígnea (18), la Edafología (17), la Geofísica (17), la Geología de los Hidrocarburos (16), la Geoquímica (13), la Volcanología (15). El resto de los artículos se reparte entre muchos rubros como Libretos-guía, Legislación Minera, Gemología, Historia de la Geología de México, Geología Marina, Geotermia, Astronomía, Geología Ambiental con 9 artículos desde 2013, Paleoclimatología (8 artículos publicados en 2010). Finalmente 20 artículos fueron clasificados como "varios".

A pesar de una producción errática de 1904 a 2000, el Boletín de la Sociedad Geológica Mexicana ha sido un testigo de la actividad geológica de México y de la evolución de las ideas. Gracias a la recuperación de su periodicidad a partir de 2000, y de su regularidad a partir del 2006, el Boletín se ha vuelto nuevamente un foro importante de la difusión de las Ciencias de la Tierra en México. 


\title{
Breve historia del Boletín de la Sociedad Geológica Mexicana
}

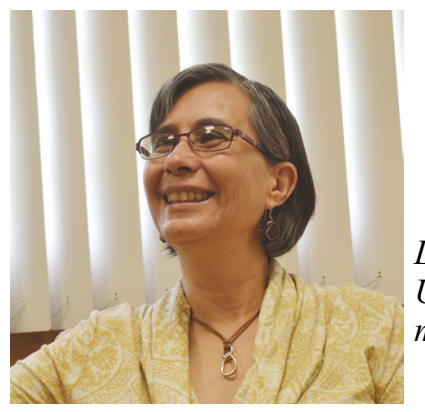

\author{
Elena Centeno García
}

La Dra. Elena Centeno García es Investigadora Titular B del Instituto de Geología de la Universidad Nacional Autónoma de México, entidad de la que es directora desde 2010. Es miembro del Comité Editorial del Boletín de la Sociedad Geológica Mexicana desde 2005.

Felicitaciones a todos los autores, editores, revisores y personal de apoyo del Boletín de la Sociedad Geológica Mexicana en su 110 aniversario. El BSGM, además de ser uno de los medios de comunicación más antiguos en América, es también de las revistas científicas pioneras en México. Su primer número, publicado en el año de 1905, incluye además de los estatutos de la SGM, la guía a las dos primeras de excursiones organizadas por la sociedad, cuyo autor fue el Ing. Ezequiel Ordóñez, quien por cierto, fuera el geólogo que determinó la ubicación del primer pozo petrolero productivo en México, en el año de 1903. Las excursiones tuvieron como objetivo los cráteres de Xico, en Chalco y las canteras de San Lorenzo Totolinga y Echegaray, ambos lugares ubicados en el Valle de México y que actualmente se encuentran cubiertos por las construcciones de la mancha urbana de la gran metrópoli. Este primer número del boletín, incluye 4 artículos sobre yacimientos minerales, uno más del mismo Ordóñez sobre el Cofre de Perote, un artículo sobre paleontología de Böse y una reseña del desarrollo de la Geología en México, escrita por José G. Aguilera. En todos los artículos de este primer número destaca claramente la influencia del naturalismo movimiento literario de la época y que se caracterizó por descripciones muy detalladas de lugares, personajes y situaciones, aunque se nota también la influencia del romanticismo mexicano en la narrativa, por ejemplo, de Don José G. Aguilera, de quien transcribo las cuatro primeras líneas: "La geología mexicana puede decirse que nace, de una manera informe é innominada, en las ideas que, transmitidas por la tradición, fueron recogidas y conservadas en las inscripciones jeroglíficas de las razas pobladoras del Anáhuac."

En la primera época del boletín, que abarca de 1904 a 1912, fueron publicados 8 volúmenes, en ellos, los temas más importantes giran alrededor de descripciones de sitios geológicos, descripciones de minas y yacimientos minerales, descubrimientos paleontológicos y las primeras reseñas sobre temas petroleros. Destacan por ejemplo, la descripción de los cristales gigantes de Naica, Chihuahua, el descubrimiento del Triásico de Zacatecas, los tubos de lava del Pedregal de San Ángel, y las primeras interpretaciones de los sismogramas obtenidos en la Estación de Tacubaya, realizadas por el Ing. Camacho.
La revolución y los cambios políticos, sociales y económicos subsecuentes afectaron también a la Sociedad Geológica Mexicana, por lo que la publicación de su boletín fue suspendida de 1912 a 1936. El primer volumen de esta segunda época incluye el acta de reorganización de la Sociedad Geológica Mexicana, dos artículos de F.K. Müllerried, uno de ellos dedicado a Burckhardt, fallecido un año antes y un artículo sobre metalurgia de E. Schmitter. En esta segunda época el boletín se produce de manera irregular, en algunos años se publicaron varios números de un solo volumen y en otros no se publicó. En esta época se ve un incremento en el número de artículos relacionados a la hidrología y las aguas subterráneas. Además de una descripción de los deslizamientos de tierra de la Sierra de Juárez, la estratigrafía de Huayacocotla, y estudios geoquímicos de suelos, rocas y agua. En 1956, el Lic. A. Terrones (senador) publica un artículo sobre el significado del geólogo ante la economía nacional, y cierra su texto con una frase, que no ha perdido actualidad: “... solo me resta invocar al sentido de responsabilidad de los gobiernos y de la iniciativa privada para que, percatándose de la trascendencia de dicho esfuerzo (de los geólogos), concedan a los estudios y a la investigación geológicos la importancia que merecen..."

La tercera época inicia en el año de 1957 y culmina en el año de 2003, en su primer volumen destaca la presentación por el Lic. A. Terrones ante la Cámara de Senadores de la República Mexicana, sobre el XX Congreso Geológico Internacional, que tuvo lugar en México en 1956. Al final de su discurso destaca la importancia de la geo-hidrología, y menciona que al menos el $30 \%$ de las investigaciones se dedican a esta rama. Aunque con algunos huecos, principalmente de 1996 al 2000, ésta época del boletín registra el rápido desarrollo y diversificación de las Ciencias de la Tierra en México. Se incluyen trabajos de astronomía, geofísica de exploración, pero también están presentes los temas importantes para la economía nacional, relacionados con yacimientos minerales y metalogenia, génesis del petróleo y yacimientos de gas, con autores como J. Álvarez Jr. En los años sesentas se desarrolla el estudio de la geología marina en México y esto se ve reflejado en varios artículos publicados en el boletín sobre batimetría, 
oceanografía, sedimentología, micropaleontología y geofísica tanto del Golfo de México como del Golfo de California. La mineralogía y la geoquímica tienen un desarrollo importante, con los trabajos de F. Fabregat y L. de Pablo. En esta época se publican los primeros artículos en idioma inglés. A finales de los sesentas, se publican artículos sobre geocronología y geotermia, y aunque dominante el número de autores nacionales y que publican sobre México, inicia la aparición de los primeros artículos sobre temas geológicos de otras regiones del país. Para finales de los años setentas se publican los primeros artículos sobre tectónica y geodinámica por Z. de Cserna, F. Campa y R. Lejsec y trabajos sobre geomorfología y las primeras imágenes satelitales Landsat. También en esta década se publican numerosos trabajos sobre estratigrafía, petrología, paleontología y metalogenia. En los ochentas y noventas las publicaciones del boletín tienen un sesgo hacia la estratigrafía y paleontología, principalmente de las regiones de la Sierra Madre Oriental y del Golfo de México, sin dejar de publicar sobre otros temas.

En el 2000 surge nuevamente la publicación del boletín con una nueva perspectiva, en su artículo introductorio sobre los Avances de la Geológica Mexicana en la Última Década, publicado por A. Nieto, en el que resalta la publicación de la Carta Geológica de México en 1992, y el Mapa Tectónico de México en 1989. Este mismo volumen incluye cuatro trabajos fundamentales sobre el volcanismo Cenozoico de México, publicados por D. Morán Zenteno, J. Aranda Gómez, A. Martín Barajas, y L. Ferrari. Los textos de ellos demuestran cómo, en casi 100 años, el conocimiento geológico evolucionó de la descripción de un par de conos volcánicos (Ordóñez en el primer volumen, a la síntesis de la estratigrafía y la génesis de las mayores provincias volcánicas de México.

Si bien el paso a la modernidad se dio a finales de la tercera época, es en la cuarta que se da, a un ritmo acelerado, la internacionalización del BSGM. Es de resaltar los tres números del volumen $57 \mathrm{y}$ un número del volumen 58 , conmemorativos de los cien años de la Sociedad Geológica
Mexicana, el primero titulado Grandes Fronteras Tectónicas de México, el segundo relacionado con los aspectos históricos de la Geología Mexicana, el tercero sobre temas selectos de la Geología Mexicana y el cuarto dedicado a los depósitos minerales de México. Para el año 2007, la Geologica Society of America, de los Estados Unidos, publica un número especial para celebrar el centenario de la SGM.

A partir del año 2005 bajo la dirección del Dr. Antoni Camprubí, como editor principal, el Boletín ha logrado posicionarse como un órgano de referencia en México para el conocimiento en Ciencias de la Tierra, ha consolidado y regularizado la publicación de tres números al año con puntualidad. Además de incrementar considerablemente tanto la temática en Ciencias de la Tierra como la extensión de los artículos. Gracias a que se constituye como una revista abierta y digital, no hay limitaciones en el número y calidad de las figuras, lo que permite la inclusión de mapas, fotografías y figuras a color. Su cuerpo editorial ha buscado en los últimos diez años, un balance entre los artículos de los temas clásicos del boletín, con aquellos de ciencia básica, y es en este tiempo cuando surgen contribuciones de nuevas áreas como la geología ambiental, la edafología y la geología urbana. Actualmente, el boletín pertenece a los índices de revistas científicas más importantes, tanto nacionales como internacionales, incluye artículos en ambos idiomas, español e inglés, y los artículos son tan diversos, en temática y en ubicación geográfica, con contribuciones de más de 20 países en su última época.

Hay que reconocer el trabajo de su cuerpo editorial a través de su historia, pero en particular, al editor en jefe actual A. Camprubí, por los éxitos logrados en los últimos 10 años. No cabe duda que le espera un futuro de muchos éxitos más.

El Boletín de la Sociedad Geológica Mexicana, es y seguirá siendo un reflejo del desarrollo de las Ciencias de la Tierra en el país y un importante medio científico de comunicación nacional e internacional. ¡Felicidades en sus 110 años! 


\title{
Carta del Presidente de la Sociedad Geológica Mexicana
}

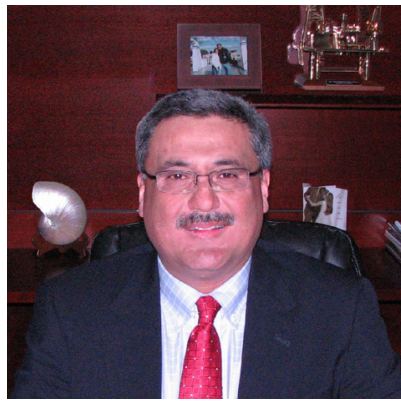

\author{
José Antonio Escalera Alcocer
}

El Ing. José Antonio Escalera Alcocer es el presidente actual de la Sociedad Geológica Mexicana (bienio 2014-2015), y fue presidente de la Asociación Mexicana de Geólogos Petroleros entre 2004 y 2006. Ha ocupado numerosos cargos en PEMEX, incluyendo el de Subdirector de Exploración en PEMEX-Exploración y Producción (PEP) entre 2007 y 2015.

En el marco del aniversario número 110 de publicar el Boletín de la Sociedad Geológica Mexicana, es buen momento para reconocer ante la comunidad geológica a quienes han contribuido a construir su gran prestigio: autores de artículos, lectores, comisión de arbitraje, y de manera especial su dirección editorial, quienes se aseguran de publicar un producto de calidad. En cada número son seleccionados con especial cuidado los artículos y autores que la integrarán para asegurar los altos estándares de calidad en presentación y contenido que le caracteriza y que es reconocida a nivel nacional e internacional.

El Boletín es una de las revistas científicas más antiguas de México. La comunidad geológica debe sentirse orgullosa por este hecho. A lo largo de su historia, primero en impresiones de papel y desde hace algunos años de manera digital, ha sido la revista por excelencia de información con el que se nutre la comunidad geológica mexicana.

Como sabemos, la Sociedad Geológica Mexicana tiene la misión de contribuir al fortalecimiento de las investigaciones geológicas para el aprovechamiento de los recursos naturales que el país demanda. También está comprometida a contribuir en el desarrollo de profesionistas relacionados con las Ciencias de la Tierra organizando la Convención Geológica Nacional y eventos de capacitación, así como publicando interesantes trabajos de investigación para divulgar los conocimientos geológicos que impactan significativamente en las áreas de investigación, educación, geología petrolera, geología minera, geotermia, geología aplicada, geohidrología y geología ambiental.

Desde su fundación en 1904, la Sociedad Geológica Mexicana, ha estado relacionada con las instituciones antecesoras del actual Instituto de Geología de la UNAM. Los primeros profesionales que integraron la Comisión Geológica Mexicana, en 1886, después Instituto Geológico Mexicano en 1888, identificaron la necesidad que tenía México de contar con instituciones científicas de prestigio, como la geológica, que aplicaran sus conocimientos en diferentes áreas y que fueran el soporte del desarrollo del país. Estos visionarios de ideas progresistas, percibieron que era necesario crear una sociedad profesional que aglutinara y que pusiera en contacto a los profesionales relacionados con la Geología, así como establecer los medios para difundir el conocimiento geológico, teniendo como uno de sus pilares más importantes este Boletín.

Si bien el primer boletín asociado al Instituto Geológico Mexicano vio la luz en el año de 1895, comienza a aparecer como Boletín de la Sociedad Geológica Mexicana en el año de 1905. A lo largo de estos 110 años se le han identificado cuatro épocas diferentes, en las que inclusive tuvo una interrupción importante de una veintena de años en la primera mitad del siglo XX. A pesar de estas interrupciones, a lo largo de su historia su misión ha tenido como constante difundir a la comunidad geológica el trabajo de investigaciones principalmente efectuadas en el Instituto de Geología de la UNAM. Hoy en día su acceso es gratuito, con lo que se asegura una amplia participación profesional para beneficio de las diversas áreas en donde puedan tener aplicación estos trabajos de investigación.

Desde mi perspectiva, es indudable el gran valor que aporta el Boletín, ya que la lectura de sus artículos sirve de guía a educadores, investigadores, profesionales de la geología aplicada y estudiantes, debido a que son una expresión genuina del avance de la Geología como área del conocimiento, adaptándose al nuevo entorno de competencia en que se encuentra hoy nuestro país.

Comentario aparte merece el hecho de la inclusión del Boletín en el Science Citation Index-Expanded, lo cual ratifica del cuidado que se tiene para la selección del material a publicar y del nivel de calidad de sus ediciones. Con este logro, el Boletín está incluido en una gran base de datos donde sólo se indexan revistas con material de alta calidad de información científica.

Lo descrito anteriormente son razones más que suficientes para un merecido reconocimiento a quienes han contribuido a lo largo de su historia, y particularmente en los últimos años, a dar al Boletín el prestigio con el que cuenta actualmente. 


\section{0 años del Boletín de la Sociedad Geológica Mexicana}

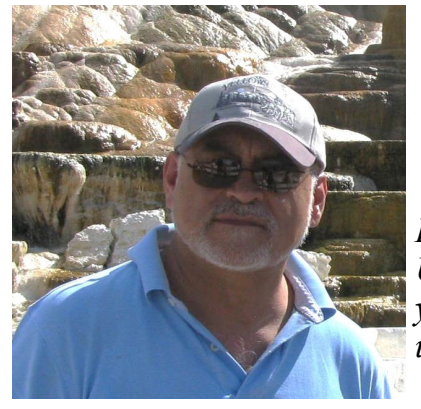

Dante J. Morán Zenteno

El Dr. Dante J. Morán Zenteno es Investigador Titular C del Instituto de Geología de la Universidad Nacional Autónoma de México, cuya Dirección ocupó entre 1994 y 2002, y es Director General de la Dirección de Asuntos del Personal Académico de la misma universidad.

Los segmentos continentales y oceánicos del territorio mexicano cuentan con una alta diversidad de rasgos geológicos. Esta diversidad se expresa en las zonas continentales como una variedad muy amplia de unidades geológicas y rasgos tectónicos que forman un patrón intrincado de relaciones espaciales y temporales configuradas durante los últimos 1800 millones de años. El entorno oceánico del territorio continental mexicano despliega también innumerables rasgos tectónicos y volcánicos que son la expresión de procesos geodinámicos en los que convergen cinco límites de placas tectónicas. A esta complejidad de la geología mexicana corresponde también una alta diversidad de recursos geológico-económicos formados en entornos, tiempos y escenarios muy variables.

El conocimiento sobre la riqueza geológica de nuestro país ha avanzado significativamente. Si bien existen todavía regiones inexploradas y problemas científicos con un intenso nivel de debate, sobre todo si se compara con el conocimiento refinado que se tiene de otras regiones del mundo, los estudios geológicos en México tienen ya una larga tradición. Sus raíces institucionales se remontan al Real Seminario de Minería, fundado en 1792, a partir del cual se desarrollaron históricamente otras instituciones de gran relevancia, como la Escuela de Ingenieros y el Instituto Geológico de México, en el siglo XIX, y posteriormente el Instituto de Geología de la UNAM y el Consejo de Recursos Minerales. A partir de la segunda mitad del siglo XX se establecieron muy diversas instituciones universitarias y de investigación que han dado un fuerte impulso al conocimiento del entorno geológico de nuestro país. Paralelamente, las instituciones y empresas encargadas de la localización, estudio y explotación de recursos geológicos de México, como el petróleo, los yacimientos minerales y los recursos hídricos, han contribuido con datos, descubrimientos e interpretaciones que han sido fundamentales para el avance de las Geociencias mexicanas. Asimismo, las contribuciones de grupos científicos y de expertos de otros países han jugado un papel significativo en la construcción del conocimiento sobre el entorno geológico de nuestro país.

Uno de los factores fundamentales del desarrollo de la larga tradición geológica de México ha sido la Sociedad
Geológica Mexicana. Desde su establecimiento en 1904, esta agrupación científica y profesional ha contribuido de muy diversas maneras al progreso de la ciencia geológica en nuestro país. Su testimonio documental más relevante es, sin duda, el Boletín de la Sociedad Geológica Mexicana. Los trabajos reunidos en sus 67 volúmenes, a partir de 1904, ofrecen un panorama de la evolución de conceptos, marcos teóricos y datos sobre la estratigrafía, el registro paleontológico, la tectónica y los recursos geológicos de nuestro país. El Boletín guarda el testimonio de cientos de autores que dejaron en sus publicaciones, observaciones, descubrimientos y reflexiones sobre el intrincado entorno geológico de nuestro territorio. En sus páginas se pueden seguir el colapso de viejas ideas y el surgimiento de nuevos paradigmas, descripciones detalladas e hipótesis provocadoras, pero también errores y especulaciones. También se puede observar la evolución del pensamiento científico de los actores más importantes.

En contribuciones del Boletín de la Sociedad Geológica Mexicana se observa la manera en que la comunidad geológica de México fue respondiendo a los cambios en los paradigmas prevalecientes en cada época. Así, en las contribuciones anteriores a la década de los 80 s, se reconocen referencias abundantes a la teoría antigua del geosinclinal para explicar los rasgos orogénicos de México y posteriormente se observa con claridad el cambio hacia los conceptos de tectónica de placas. También se puede observar de qué manera, a través de análisis modernos y fechamientos isotópicos, evolucionan los conceptos de un zócalo cristalino antiguo y continuo hacia la idea de muy diversos segmentos cristalinos que forman un mosaico de terrenos que evolucionaron en muy diversos entornos paleogeográficos y tectono-térmicos y también en diversas épocas.

Con el tiempo, el propio Boletín ha evolucionado hacia esquemas editoriales más modernos y exigentes. El panorama científico internacional ahora plantea la necesidad de dar a conocer los avances científicos de una forma documentalmente organizada y con garantías de rigor en la evaluación de los trabajos publicados. Adicionalmente, las publicaciones científicas periódicas requieren del cumplimiento de estándares internacionales para acceder 
a índices bibliográficos y sistemas de referenciación. De esto depende que una revista pueda ser consultada y citada en cualquier lugar del mundo. El Boletín no se ha rezagado en este rubro y actualmente forma parte de los principales índices bibliográficos internacionales. Las contribuciones publicadas pueden ser abiertas y leídas gratuitamente por cualquier persona. Gracias a esto, la presencia del Boletín ha crecido y se ha convertido en uno de los foros fundamentales para el análisis, la reflexión y la discusión geocientífica en México.

Los artículos publicados a nivel mundial sobre la geología de México son abundantes. Esto se debe a que nuestro territorio tiene rasgos geológicos excepcionales y emblemáticos que han llamado la atención de muchos grupos científico en el mundo. De acuerdo a las evaluaciones sobre el impacto de los trabajos geocientíficos publicados en México realizadas por la organización SCImago Journal and Country Rank, las Geociencias mexicanas tienen más influencia mundial que muchas otras disciplinas en México. Esto revela que la comunidad geocientífica mexicana ha desarrollado un dinamismo que es menester, nos solo sostener, sino impulsar aún más para beneficio de la sociedad mexicana y de las futuras generaciones. En la construcción de la tradición geológica de México y el desarrollo de una comunidad geológica cada vez más dinámica, el Boletín de la Sociedad Geológica Mexicana representa un símbolo de esfuerzo, persistencia y búsqueda de excelencia que hay que celebrar. 


\section{El Boletín de la Sociedad Geológica Mexicana como revista científica}

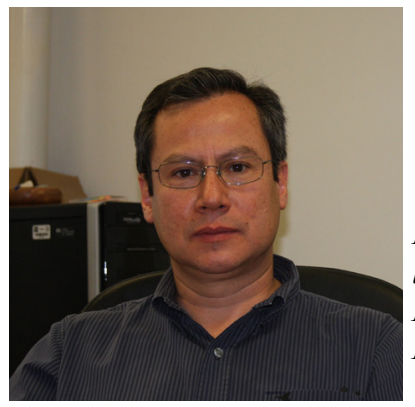

Ángel F. Nieto Samaniego

El Dr. Ángel F. Nieto Samaniego es Investigador Titular C del Centro de Geociencias de la Universidad Nacional Autónoma de México. Fue Presidente de la Sociedad Geológica Mexicana en el bienio 1997-1998, y Editor en Jefe del Boletín de la Sociedad Geológica Mexicana hasta 2005.

He tenido la fortuna de ser miembro de la Sociedad Geológica Mexicana desde casi veinte años atrás y tuve el gusto de participar en el comité editorial de su Boletín. Esta publicación centenaria tiene escritas en sus páginas muchas de las bases del conocimiento geológico de México. Con 110 años de publicación, el Boletín representa un acervo muy extenso y, como es natural, ha atestiguado el cambio de paradigmas y la llegada de nuevas tecnologías a las ciencias de la Tierra. También, y no menos importante, ha logrado cambiar de una edición artesanal a principios del siglo $\mathrm{XX}$, a un formato totalmente electrónico a principios del siglo XXI.

Me tocó participar en dos importantes momentos: el número especial "Avances de la Geología Mexicana en la última década" publicada en el año 2000 y el volumen conmemorativo del centenario de la Sociedad Geológica Mexicana, el cual se publicó en Español en el nuestro Boletín, y una selección de esos trabajos aparecieron en idioma inglés como un Special Paper de la Geological Society of America. La publicación conmemorativa del centenario reunió los esfuerzos de la comunidad geocientífica nacional para reflexionar sobre el estado del conocimiento de la geología de nuestro país y constituyó la culminación de una etapa en la vida del Boletín. A partir de entonces tuvo lugar una profunda reestructuración y cambio de políticas editoriales, las cuales permitieron al Boletín configurarse paulatinamente como una publicación moderna, electrónica, de distribución mundial por medio de Internet y que se encuentra incluida en los índices de revistas científicas más prestigiados del mundo. Esta nueva cara del Boletín tiene implicaciones muy importantes que me invitan a hacer una reflexión:

El Boletín vuelve a ser una revista preponderantemente de carácter científico y se inserta en la comunidad internacional como una de las dos más importantes publicaciones mexicanas sobre Geología. El éxito que se logre en el ámbito internacional dependerá de la acogida que tengan, por parte de la comunidad científica, las investigaciones allí publicadas, lo a su vez depende de la calidad y profundidad de las investigaciones que se publiquen. No tengo duda de que en la actualidad existe en México una comunidad de investigadores de las ciencias de la Tierra suficientemente grande y con un nivel suficientemente alto para mantener con éxito a nuestro Boletín. También pienso que los datos e ideas que allí se publiquen nutrirán a la sociedad con información muy valiosa en los ámbitos educativo, social y económico. Por otra parte, un sector de nuestra comunidad labora en numerosas organizaciones privadas y gubernamentales, y se encuentra realizando trabajo fuera del ámbito científico, más bien están dedicados a la aplicación de conocimiento tecnológico con fines prácticos, ya sea enfocados a la exploración o explotación de recursos naturales, a la evaluación, prevención o mitigación de desastres naturales, o bien a dar soporte a trabajos ingenieriles de gran envergadura. Esa comunidad requiere también un foro en donde publicar la información pertinente a cada uno de sus campos de trabajo, pero típicamente sus publicaciones no llenan los requisitos de una publicación científica, lo cual ha hecho que no les sea posible publicarlos en las revistas científicas internacionales. Ahora, con los nuevos estándares del Boletín, difícilmente les será posible hacerlo allí. Esta separación de esa parte de nuestra comunidad no es necesariamente negativa, lo veo como una "especialización" de nuestro Boletín, ya que le permite definir mejor su identidad como un foro científico y deja a otras revistas la tarea de acoger las publicaciones de corte más tecnológico. Ese tipo de revistas ya existen, podría mencionar solo a manera de ejemplo GEOMIMET y Geotermia; mi reflexión es que, con esta paulatina separación del Boletín hacia ámbitos más académicocientíficos, se hace necesario el fortalecimiento de dichas publicaciones para que ambas partes se vean beneficiadas $y$, de esa manera, en lugar de generar una sensación de pérdida de espacios para publicar, se tenga una segmentación o especialización de los foros para llevar a cabo la publicación de nuestros trabajos. Los autores potenciales de trabajos de corte tecnológico son muy numerosos, sin embargo es limitado el número de ese tipo de trabajos que se publican. Es común que el rechazo de artículos técnicos en foros científicos cree desánimo en los autores, pues se confunde el rechazo de un manuscrito por no ser adecuado para una publicación científica, con un cuestionamiento a la calidad del trabajo, pero en realidad lo que ocurre es que el foro donde se intenta publicar no es el correcto. Pienso que esta 
es una buena oportunidad para diferenciar más claramente los distintos tipos de publicaciones; también me parece que con la correcta identificación del enfoque de cada revista, los autores se verán beneficiados.

Veo en esta nueva etapa del Boletín un futuro prometedor, y la comunidad geocientífica de México seguramente agradecerá contar con una nueva revista con los más altos estándares científicos y en donde podrá, si así lo prefiere, publicar en idioma español 


\section{Mis experiencias e impresiones sobre el desarrollo académico del Boletín de la Sociedad Geológica Mexicana}

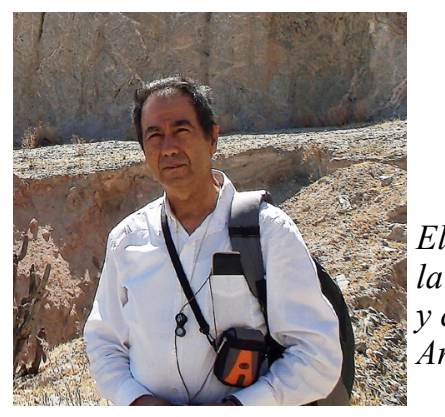

Fernando Ortega Gutiérrez

El Dr. Fernando Ortega Gutiérrez es investigador emérito del Instituto de Geología de la Universidad Nacional Autónoma de México, cuya Dirección ocupó entre 1986 y 1994, y con 45 años de antigüedad académica. Es Senior Fellow de la Geological Society of America, de cuyo Consejo Directivo formó parte.

Como geólogo investigador desde 1968 en el Instituto de Geología y su antecesor el Instituto Geológico de México, en cuyo seno surgió la Sociedad Geológica Mexicana y su primer Boletín en 1904, aprovecho esta magnífica oportunidad para reflexionar sobre la huella tan profunda que ha dejado a su paso esta publicación científica de las Geociencias en el desarrollo de la Ciencia en México. Tres épocas han marcado su devenir histórico, la primera con sus ocho volúmenes contiene contribuciones de personajes legendarios de la Geología mexicana como Ezequiel Ordóñez y José Guadalupe Aguilera, el primero con sus múltiples contribuciones sobre aspectos tanto de la geología básica (los cráteres del Xico y el volcán Cofre de Perote) como económica, y el segundo con su extraordinaria reseña sobre el estado del arte de la Geología de México en esa época. También son dignos de mención los trabajos de Teodoro Flores o Emilio Böse sobre los "criaderos" minerales o los fósiles de regiones varias del país.

Desde mi perspectiva, durante la vigencia de su segunda época, me parece justo señalar que el papel mas relevante que ha jugado el Boletín de la Sociedad Geológica Mexicana es el haber sido el vehículo para la presentación pionera de documentos básicos y sintéticos sobre la Geología de cualquier país, como fueron las primeras cartas de provincias tectónicas de México (M. Álvarez) y carta gravimétrica (A. de la O Carreño), ambas en 1949, más tarde la metalogénica (C. Castillo-Tejero, 1956) y, por último, la fisiográfica (1961), también por Manuel Álvarez. Durante esta segunda época es un verdadero placer constatar y disfrutar el conocimiento logrado por documentos clásicos sobre la Geología de México, los cuales prevalecen después de muchas décadas desde su publicación, como fueron los de R. Imlay sobre el sistema Jurásico de México (1953), de $\mathrm{H}$. Larios por sus trabajos pioneros en la naciente disciplina de la Geoquímica (1954), o bien sobre los primeros fechamientos absolutos que se hicieron en México por métodos radiométricos (1966), sin olvidar algunos documentos que se adelantaban a su época, como los del Z. de Cserna sobre la tectónica del sur de México (1967), M. Tardy sobre la Sierra Madre Oriental (1972) y F. Mooser (1987) sobre la Faja Volcánica Transmexicana.

La segunda, junto con esta tercera época vigente hoy de la historia del Boletín, han sido igualmente prolíficas en la presentación de trabajos científicos llamados a ser los clásicos de la Geología mexicana del siglo presente; en particular, todos aquellos que se publicaron al iniciarse el siglo sobre los avances del conocimiento de nuestros principales sistemas geológicos en el 2000, o los emanados de sus volúmenes conmemorativos 57 y 58 en 2005 y 2006, dando a conocer aquí síntesis estupendas sobre las grandes fronteras tectónicas de México, sus provincias volcánicas mas relevantes y sobre la naturaleza (tipología) de sus yacimientos minerales.

Con su nueva estrategia de desarrollar la publicación de volúmenes especiales sobre temas centrales para el bienestar de la sociedad mexicana se debe mencionar su más reciente número sobre geología del Valle de México, haciendo así honor a una tradición del Boletín que desde sus primeros volúmenes atendió sin regateos problemas fundamentales sobre el conocimiento de las condiciones geológicas de la Cuenca o Valle de México, claramente reflejados en sus riesgos y peligros derivados de su cercanía a regiones sísmica y volcánicamente activas, así como sujeta a las consecuencias negativas del crecimiento desmesurado de la ciudad, como han sido la contaminación de sus ecosistemas, el hundimiento del suelo y el agotamiento de sus acuíferos.

En esta reflexión no podría ignorar un hito en el desarrollo académico del Boletín de la época actual, el cual fue su admisión en 2014 en el Science Citation Index manifestando un índice inicial de impacto de 0.432 que lo coloca entre las primeras revistas geocientíficas de México.

Termino esta reflexión señalando dos aspectos del Boletín que, a corto plazo y a mi juicio, podrían implementarse con el único afán de incrementar su prestigio y trascendencia más allá de nuestras fronteras: primero, solicitar contribuciones sobre temas avanzados de investigación en Geociencias a líderes nacionales e internacionales que por su circunstancia particular hayan construido una parte sustancial de su carrera científica nutriéndose de los espléndidos laboratorios naturales que brinda la Geología mexicana; segundo, profundizar el rigor de los arbitrajes para distinguir con claridad las contribuciones que avancen mejor el conocimiento científico en general, o bien que trasciendan por su aplicación a la solución de problemas derivados del modo como funciona e interacciona la naturaleza física con el entorno de nuestra civilización. 


\section{Indicadores de producción e impacto del Boletín de la Sociedad Geológica Mexicana}

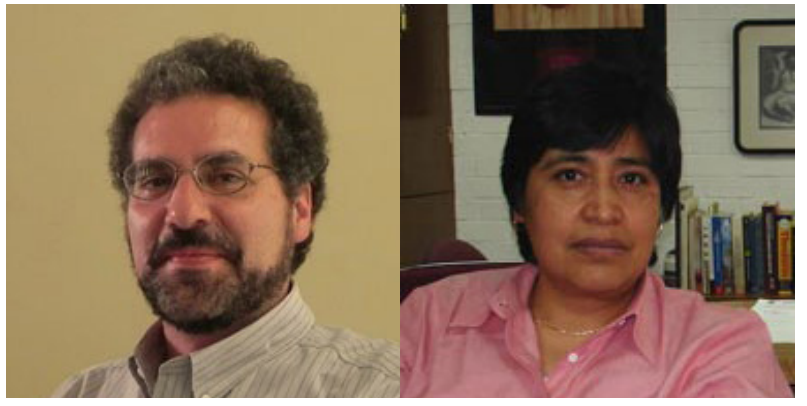

La inclusión de la revista Boletín de la Sociedad Geológica Mexicana (BSGM) en índices y bases de datos científicas, en particular, aquellas que pueden ser referidas como bases de datos "bibliométricas" en tanto que ofrecen indicadores cuantitativos de las revistas, nos permite conocer aspectos del comportamiento de la revista revelados por el "rastro" bibliométrico que ésta ha dejado a su paso: su diálogo con la comunidad a la que está dirigida, su exogeneidad institucional y geográfica, su impacto medido en el sentido estricto de las citas recibidas así los tiempos de recepción y asimilación del conocimiento difundido por la revista. No obstante, a pesar de que el BSGM es una revista centenaria, su inserción en los índices bibliométricos es relativamente reciente, puesto que nos remite a la década pasada. En este breve recuento se exponen algunos indicadores reportados por los índices Periódica-Biblat ${ }^{1}, \mathrm{SciELO}^{2}$, SciELO Citation Index-WoS ${ }^{3}$, Web of Science (WoS) y Scopus ${ }^{4}$. Por otra parte, hemos subdividido los indicadores aquí presentados en dos: indicadores de producción y los indicadores de citación. Los primeros reflejan la representación institucional y nacional en los artículos publicados por la revista y que tienen como elemento de referencia la institución y el país de la institución a la que está adscrito el autor y/o coautor de cada artículo; los segundos están basados en la citación recibida por la revista por parte del universo de revistas incluido (indizado) en la base de datos fuente.

\author{
Antonio Sánchez Pereyra, Oralia Carrillo Romero
}

El Dr. Antonio Sánchez Pereyra y la Ing. Oralia Carrillo Romero son miembros del equipo editorial de SciELO-México, laborando en la Dirección General de Bibliotecas de la Universidad Nacional Autónoma de México.
La Gráfica 1 muestra la aportación preponderante de investigadores adscritos a la Universidad Nacional Autónoma de México dentro del conjunto de trabajos publicados en el período 2000 - 2015 y en los que se suman un total de 210 instituciones y organismos de diverso tipo (en la gráfica se muestran las 12 instituciones con mayor número de artículos). La Gráfica 2 presenta esta información agregando las instituciones por su pertenencia nacional y en porcentajes, mostrando que el $74 \%$ de los artículos publicados es de autores y coautores afiliados a instituciones mexicanas. La concentración institucional y nacional de artículos publicados es al mismo tiempo un asunto deseable de superar mediante la captación de mayores porcentajes de contribuciones del extranjero, lo que permitiría una mayor internacionalización de la revista, así como la expresión de una publicación nacional orientada a exponer los avances y los temas de investigación planteados en el país.

La Gráfica 3 resume el número de documentos (cobertura) del BSGM indizados en cuatro bases de datos bibliométricas y las citas totales recibidas. La indización de la revista en Scopus y SciELO (y, por tanto, también de SciELO CI) data de 2008; por otra parte, recién en 2014 la revista fue aceptada en WoS y la indización de documentos en esta base inicia en 2012. Las diferencias en los números de documentos indizados reportados por cada sistema se deben a que tienen diferentes períodos de cobertura e indización, y a que, en el caso de SciELO, sólo

\footnotetext{
${ }^{1}$ Periódica (Índice de Revistas Latinoamericanas en Ciencias) es una base de datos cuyo objetivo es difundir la literatura científica publicada en América Latina y el Caribe, por lo que sólo indiza revistas editadas en esta región. La base de datos es de acceso libre en http://periodica.unam.mx. Por otra parte, Biblat (http://biblat.unam.mx) es una plataforma para la generación y consulta de indicadores bibliométricos de las revistas indizadas en Periódica.

${ }^{2}$ SciELO (Scientific Electronic Library Online) es una red de bibliotecas de revistas científicas conformada por colecciones nacionales de quince países: Argentina, Bolivia, Brasil, Chile, Colombia, Costa Rica, Cuba, España, México, Paraguay, Perú, Portugal, Sudáfrica, Uruguay y Venezuela. SciELO cumple un doble cometido: la publicación en línea y en acceso abierto de los contenidos en texto completo de los artículos y generar indicadores bibliométricos que reflejan el impacto medido en citas por parte de revistas que conforman estas quince colecciones, esto es, representa una medida de impacto regional toda vez que contabiliza las citas provenientes de revistas de la región (con excepción de Sudáfrica).

${ }^{3}$ SciELO Citation Index (SciELOCI) es una base de datos con información de las revistas indizadas en la red SciELO, integrada en el sistema de base de datos de Web of Science (WoS). En este sentido, puede decirse que SciELOCI es una sub-base de SciELO y una sub-base de WoS: incorpora un subconjunto de las revistas de toda la red SciELO y es una base de datos integrante del conjunto de bases de datos de WoS. La utilidad de SciELO CI reside en que contabiliza las citas que recibe cada revista provenientes del conjunto global de bases de datos de WoS, incluyendo la llamada Colección Principal (Core Collection), al mismo tiempo que aporta a este sistema la información y las citas contenidas en un mayor número de revistas latinoamericanas. Esto quiere decir que SciELO CI ofrece la sumatoria del impacto medido en citas tanto de la región latinoamericana como del resto del mundo.

${ }^{4}$ Web of Science (propiedad de la empresa Thomson-Reuters) y Scopus (propiedad de la empresa Elsevier) son las bases de datos bibliométricas multidisciplinarias con mayor cobertura y sus indicadores son referencia mundial para la valoración cuantitativa del desempeño de las revistas científicas.
} 


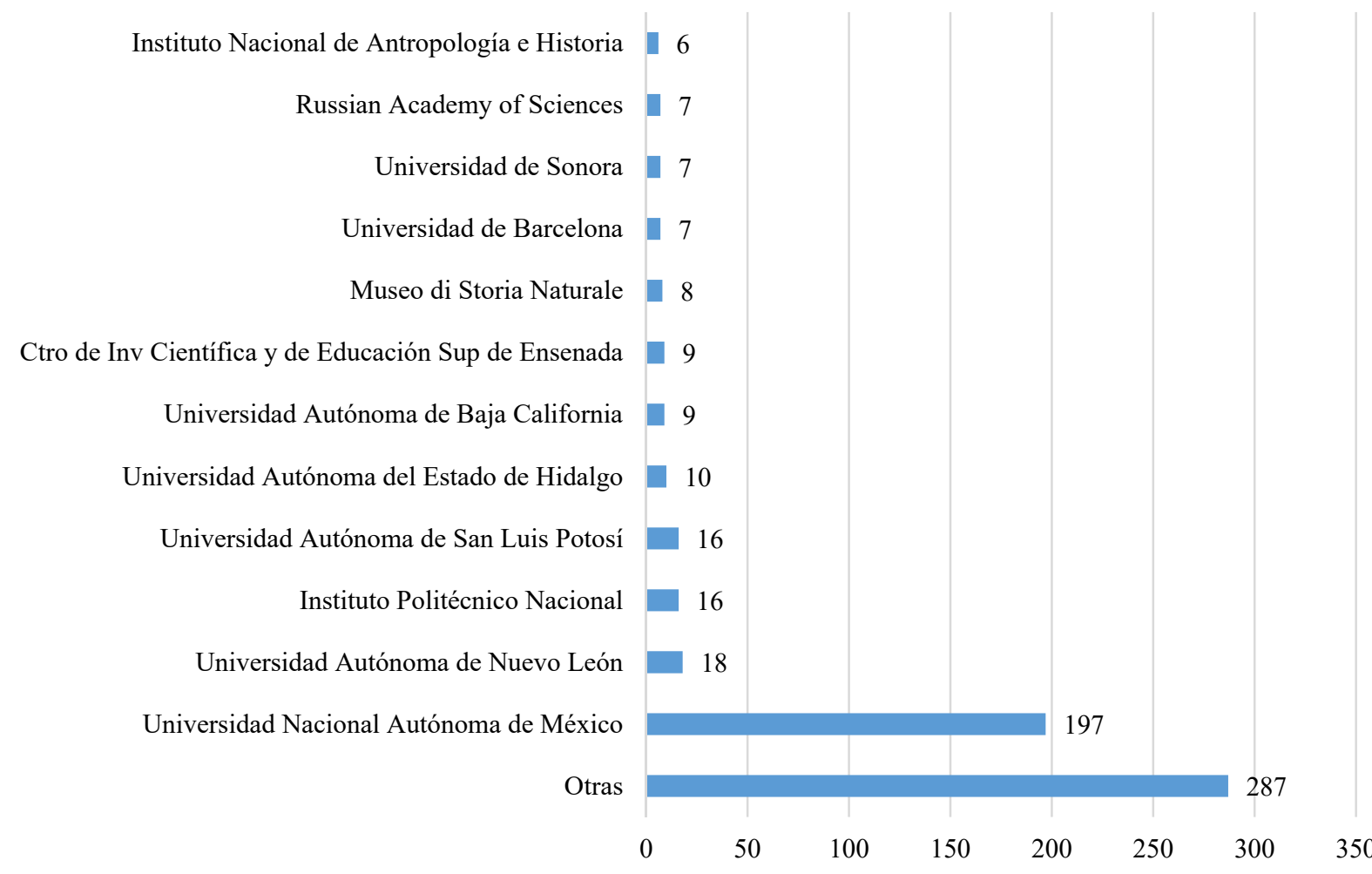

Gráfica 1. Distribución de artículos publicados en el BSGM según la institución de afiliación del autor. Periodo: 2000 - 2015.

Fuente: Periódica-Biblat $<$ http://biblat.unam.mx/es/frecuencias/revista/boletin-de-la-sociedad-geologica-mexicana/institucion>.

Cobertura en Periódica-Biblat (a nov. 2015): <http://biblat.unam.mx/es/frecuencias/revista/boletin-de-la-sociedad-geologica-mexicana/anio>.

Nota: no se incluyen datos de 2004, ya que no se ha indizado la información de este año en la base Periódica-Biblat.

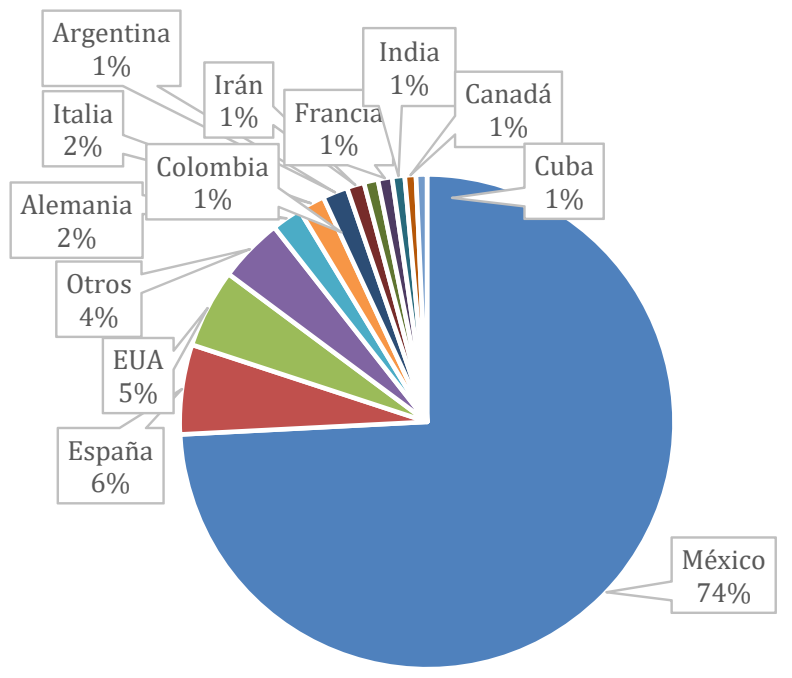

Gráfica 2. Distribución de artículos publicados en el BSGM según el país de la institución de afiliación del autor. Periodo: 2000 - 2015 (en porcentajes). Fuente: Periódica-Biblat <http://biblat.unam.mx/es/indicadores/ productividad-exogena/disciplina/geociencias $>$.

Cobertura en Periódica-Biblat (a nov. 2015): <http://biblat.unam.mx/es/ frecuencias/revista/boletin-de-la-sociedad-geologica-mexicana/anio>.

Nota: no se incluyen datos de 2004, ya que no se ha indizado la información de este año en la base Periódica-Biblat. se consideran los documentos citables, esto es, artículos y no las reseñas bibliográficas ni las editoriales. En cuanto a las citas recibidas, Scopus registra una mayor cantidad mientras que en WoS la citación registrada es menor, pero debe considerarse que en esta base la revista es de reciente ingreso y el número de años de colección registrados es menor. En SciELO el número de citas recibidas registradas es menor, lo que responde a que el universo de revistas en este sistema es mucho menor a los dos anteriores, al mismo tiempo que es menor el número de títulos de la especialidad. Por su parte, en SciELO CI se obtiene la suma de citas recibidas por la revista provenientes tanto de las revistas indizadas en WoS, como en SciELO.

La Gráfica 4 muestra el comparativo de los indicadores de impacto de las bases de datos; estos indicadores se basan en la citación recibida por la revista aunque su procedimiento de cálculo es diferente: en el caso del Factor de impacto (FI) de WoS y SciELO, el cálculo se basa en la relación entre las citas recibidas y los artículos publicados por la revista en una ventana de tiempo de 2 años, mientras que SCImago Journal Rank (SJR) pondera con un valor mayor la citas recibidas de aquellas revistas de mayor prestigio y se basa en una ventana de citación de tres años. 


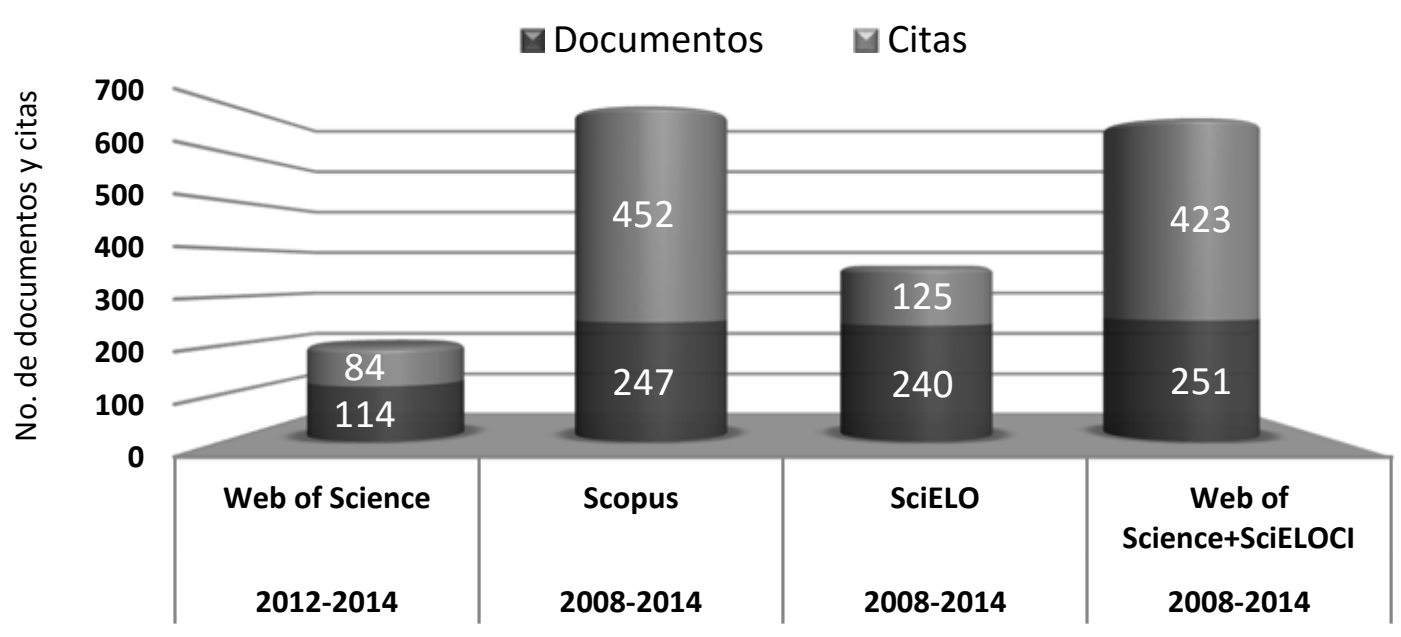

Gráfica 3. Cobertura del BSGM en bases de datos.

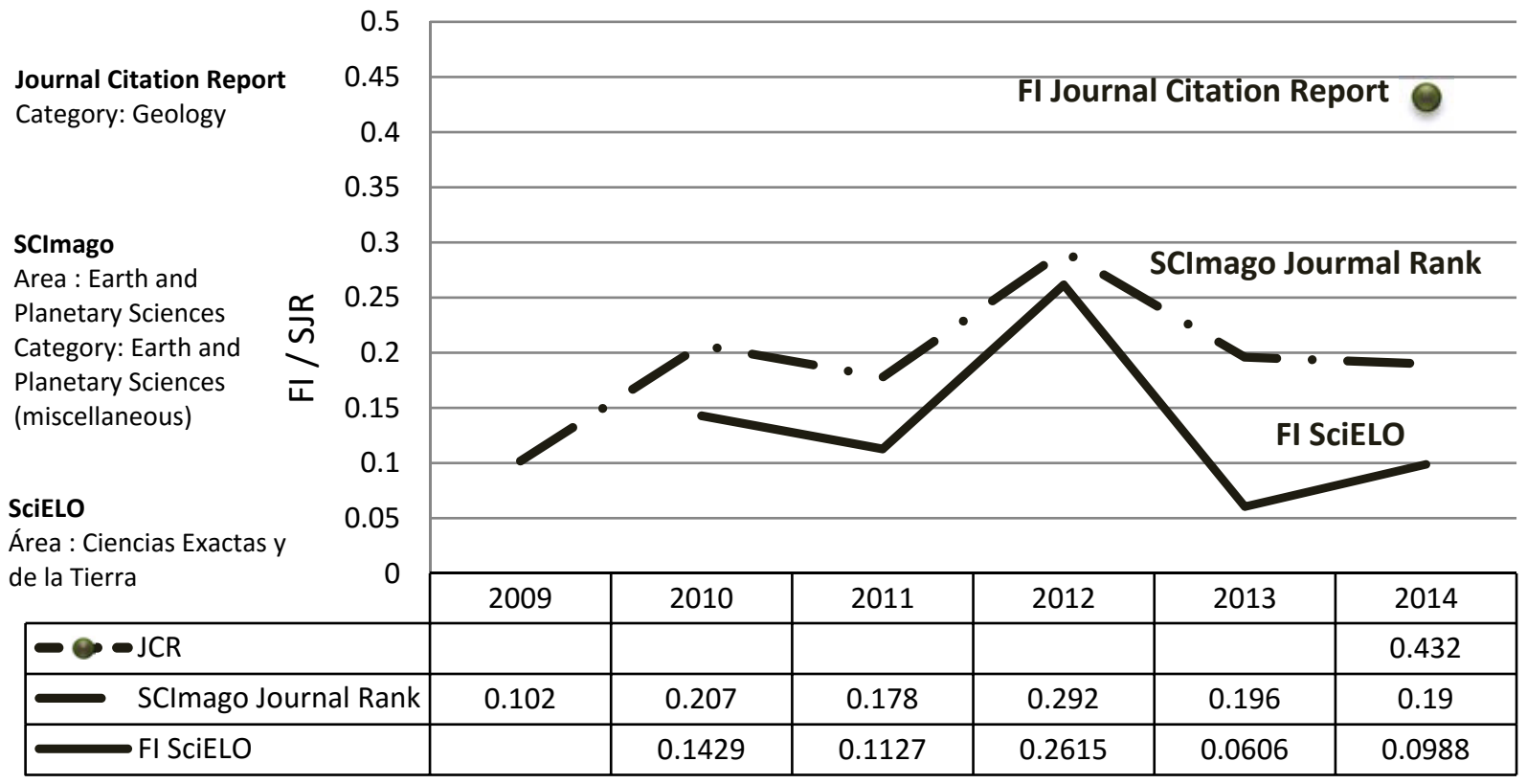

Fuente: Web of Science, SCImago, SciELO.

Gráfica 4. Indicadores de impacto

Se observa que la tendencia en Scopus y SciELO tiene un comportamiento semejante aunque con valores absolutos diferentes. En 2014, el BSGM participó por primera vez en el reporte de FI en WoS con un valor notablemente mayor que el de las otras dos bases de datos. Según dicho reporte (Journal Citation Reports 2014) la revista obtuvo un FI de 0.432 y ocupó el lugar 40 de un total de 46 revistas que conformaron en ese año la categoría Geology, ubicándose en el $4^{\circ}$ cuartil. En el reporte de SCImago de 2014 el valor de SJR obtenido es de 0.190 , lo que posicionó a la revista en el lugar 164 de 259 revistas de la categoría Earth and Planetary Sciences (miscellaneous) en la que está clasificada y en el mismo $3^{\text {er }}$ cuartil en el que se ubicó en 2013. De acuerdo con SCImago, en 2012 el BSGM obtuvo su mejor posicionamiento $\left(2^{\circ} \text { cuartil }\right)^{5}$.

Finalmente, debe considerarse que los indicadores de impacto como el FI y SJR están basados en períodos o ventanas de tiempo orientados a medir la velocidad o actualidad en la que los artículos de la revista son citados. No obstante, los documentos científicos poseen otra

\footnotetext{
${ }^{5}$ Fuente: SCImago $<$ http://www.scimagojr.com/journalsearch.php?q=15600154707\&tip=sid \&clean=0 $>$.
} 
temporalidad digna de ser considerada y que suele ser ignorada por la centralidad otorgada al FI y el SJR. Existen otros indicadores, como el de Vida Media de las citas recibidas, en el que se contempla el número promedio de años en los que se acumula el $50 \%$ de las citas recibidas por la publicación. Este indicador es proporcionado por WoS y SciELO, y como se muestra en la Tabla 1, según el único año reportado por WoS, el $50 \%$ de las citas recibidas por el BSGM en 2014 está referido a los documentos publicados por la revista entre 2010 y 2014, esto es, en un período de cinco años; según el reporte de vida media proporcionado por SciELO, el BSGM recibe citas en períodos de tiempo que van desde los tres a nueve años. Esto quiere decir que la citación recibida por la revista no se agota en el período de 2 y 3 años considerados por el FI y SJR.
Tabla 1. Vida media citada del BSGM.

\begin{tabular}{ccc}
\hline & $\begin{array}{c}\text { Web of Science } \\
\text { (años) }\end{array}$ & $\begin{array}{c}\text { SciELO } \\
\text { (años) }\end{array}$ \\
\hline 2008 & & 3.77 \\
2009 & & 9.57 \\
2010 & & 5 \\
2011 & & 6.58 \\
2012 & & 7.06 \\
2013 & & 4.91 \\
2014 & 5.6 & 7.5 \\
\hline
\end{tabular}

Fuente: $<$ http://statbiblio.scielo.org//stat_biblio/index.php?no=07.xml\&s tate $=07 \&$ lang $=$ es\&issn $=1405-3322>$.

Fecha de consulta: Noviembre 18, 2015. 